\title{
ARTICLE OPEN \\ By the numbers: ratings and utilization of behavioral health mobile applications
}

\author{
Andrew D. Carlo $\mathbb{D}^{1}{ }^{1}$, Reza Hosseini Ghomi ${ }^{2}$, Brenna N. Renn (D) $^{1}$ and Patricia A. Areán ${ }^{1}$
}

Although >10,000 behavioral health applications ("apps") are currently available on the Apple and Google Play marketplaces, they have been minimally evaluated or regulated and little is known about "real world" usage patterns. This investigation combined data from online behavioral health app rating frameworks and a mobile health market research firm to identify the most downloaded apps as well as determine rating and ranking concordance between frameworks. Findings demonstrated that the most commonly downloaded apps focus on relaxation, mindfulness, and meditation skills and that they often have notably discordant reviews across rating frameworks. Our results suggest that there is a growing need for: (1) standardized behavioral health app quality and effectiveness measures, (2) up-to-date behavioral health app guidance for clinicians and consumers, and (3) evidence-based apps that incorporate revealed consumer preferences.

npj Digital Medicine (2019)2:54 ; https://doi.org/10.1038/s41746-019-0129-6

\section{INTRODUCTION}

In recent years, the development of mobile behavioral health applications ("apps") has paralleled the growth in smartphone and digital technologies. The latest estimates suggest that between 165,000 and 325,000 health and wellness apps $^{1-4}$ are now commercially available to patients, with $>10,000$ designed specifically for mental or behavioral health. ${ }^{5}$ Although a relatively small number of these behavioral health apps have undergone rigorous evaluation in controlled trials, ${ }^{6-8}$ the vast majority remain largely unevaluated ${ }^{9}$ or claim to be evidence-based primarily because they are informed by evidence-based treatments (e.g., cognitive behavioral therapy or mindfulness). ${ }^{5}$ Further, few studies have substantiated the effectiveness of behavioral health apps outside of research settings, limiting the external validity of existing empirical findings.7,10 Even when apps are evidence based, their public health impact is often curbed by poor adherence $^{11-15}$ and a lack of availability to the general public through commonly used channels, such as the Apple and Google Play marketplaces. ${ }^{16,17}$

Nevertheless, interest in digital health technologies continues to grow and more than half of mobile device users have downloaded at least one health-related $\mathrm{app}^{18}$ at some point in their lives. Owing to the lack of publicly available information on the quality or effectiveness of these apps, purchasing and downloading decisions are often made using heuristics that compel the user to quickly weigh easy-to-identify metrics or attributes, such as title, logo, price, and marketplace star ratings. ${ }^{19}$ To allow consumers to make more informed choices, a number of systematic frameworks (with and without expert reviews ${ }^{2,20-22}$ ) have been created to rate or rank health apps for different medical conditions (e.g., behavioral health disorders, sickle-cell disease, heart disease, diabetes, and asthma ${ }^{23}$ ) across a variety of dimensions, including security/privacy, evidence base, ease of use, and interoperability. ${ }^{24}$ However, to date, there is no universally accepted resource or method, ${ }^{25}$ and it remains unclear whether existing tools reach concordance on commonly rated applications.

For years, government oversight and regulation have failed to keep pace with mobile health app growth, leaving consumers potentially vulnerable to apps that claim to offer more than they can deliver. This is expected to be partially addressed in the near future with the United States Food and Drug Administration's (FDA) recently initiated Digital Health Software Precertification (Pre-Cert) Program, which will allow for the evaluation and monitoring of digital health products, including smartphone apps, from pre-market development to post-market performance. ${ }^{26}$ Although a significant regulatory step, this "opt-in" program is not expected to influence the majority of apps or developers, as most will likely continue to offer products to consumers without the involvement of the FDA. Nevertheless, it remains incumbent upon regulators to ensure that the most commonly used apps are providing high-quality and effective services to consumers.

There is a consequent emerging need to better understand "real world" behavioral health app usage. At present, little is known about which apps are most popular among consumers and whether such popularity aligns with app quality. A recent investigation examined several characteristics of common behavioral and medical health apps, including World Health Organization (WHO) digital intervention classification, ${ }^{27}$ marketplace rating, evidence base, developer "medical claims," and consumer costs, among others. ${ }^{17}$ Although specific apps were not identified by name, the authors noted considerable content heterogeneity, such that they were unable to make objective quality assessments. ${ }^{17}$ This raises questions about contemporary behavioral health app rating frameworks, ${ }^{2,20-22}$ as they often do not overtly acknowledge their inherent methodological subjectively. At the same time, these frameworks and expert reviews may be playing an important role in helping consumers and clinicians navigate the complex, crowded, and poorly studied behavioral health app marketplace. Therefore, it is necessary to understand how

\footnotetext{
${ }^{1}$ Department of Psychiatry \& Behavioral Sciences, University of Washington, Seattle, WA, USA and ${ }^{2}$ Department of Neurology, University of Washington, Seattle, WA, USA
} Correspondence: Andrew D. Carlo (adc42@uw.edu)

Received: 22 February 2019 Accepted: 24 May 2019

Published online: 17 June 2019 
consumer-facing frameworks with expert reviews rate commonly used apps and whether they do so consistently and rigorously.

This investigation aims to address current gaps in the literature by: (1) identifying the most downloaded and installed apps for mental and behavioral health disorders and (2) comparing the ratings of the most downloaded and installed apps from three consumer-facing, publicly available, online rating frameworks with expert reviews.

\section{RESULTS}

Behavioral health app sample

A total of 441 unique behavioral health apps remained in the final sample after removing duplicates, discontinued apps, and those only available through a web browser. Apps with $<1000$ total global downloads since first tracked were excluded from the analysis (189 from Apple and 261 from Google Play). Additionally, 48 Apple and 34 Google Play apps were excluded for having a primary focus on sleep, white noise, nature sounds, or general health/wellbeing). See Fig. 1 for a detailed flow chart on app inclusions and exclusions.

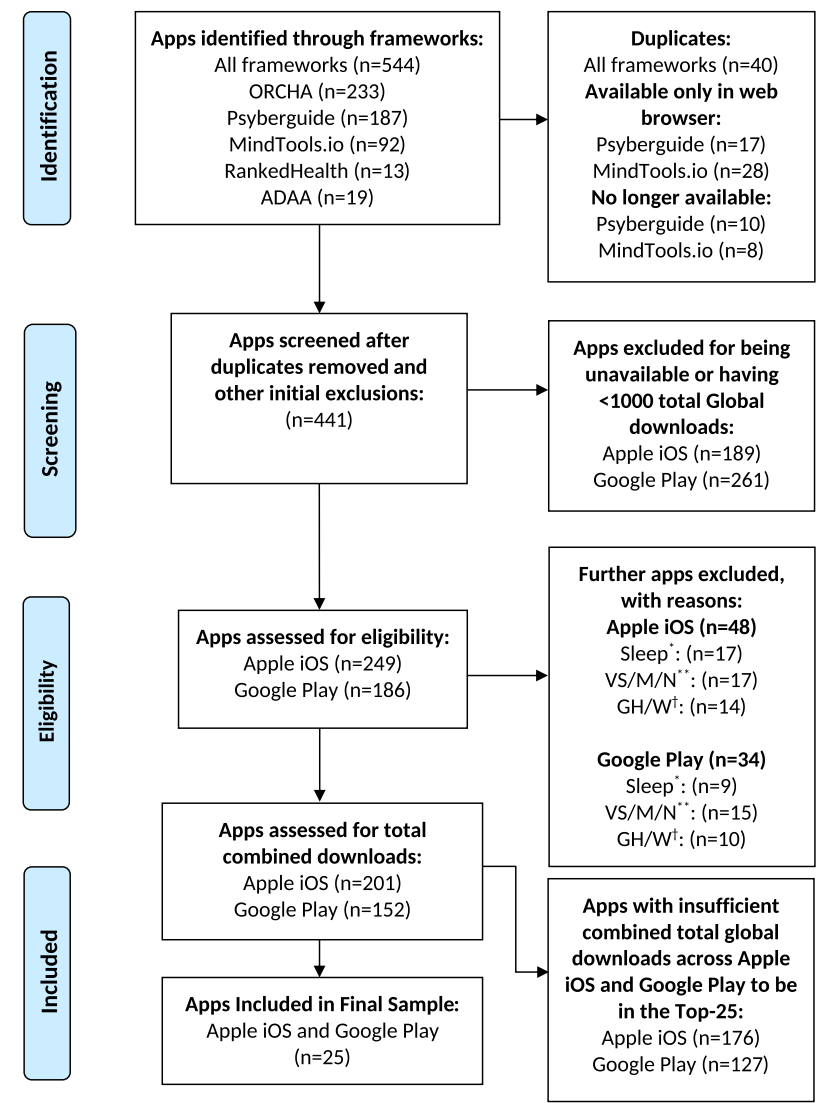

Fig. 1 Behavioral health app inclusion and exclusion flow chart. *Sleep apps were excluded if they were not designed to treat a behavioral health disorder or not promoting a specific behavioral health treatment or technique (e.g., mindfulness, meditation). **Apps with a primary focus on nature/soothing visual scenes, music/sounds, or noise/white noise were excluded, although some behavioral health-focused apps did include these features as part of their treatment package. †Apps with a primary focus on general health or wellness were excluded, although some behavioral healthfocused apps did include these features as part of their treatment package
Downloads and utilization

Table 1 lists the 25 most popular behavioral health apps based on total combined Apple App Store (iOS) and Google Play Store (Android) global downloads. Total global downloads ranged from a high of $42,300,000$ for Peak-Brain Training ${ }^{28}$ to 872,600 for Calm Harm. ${ }^{29}$ Overall, apps featuring meditation, mindfulness, and relaxation skills were most common, comprising 19 of the top 25 (76\%). The two most downloaded apps, however, were cognitive training applications (Peak-Brain Training ${ }^{28}$ and Lumosity ${ }^{30}$ ). Three of the top 25 (12\%) offered peer support, while $4(16 \%)$ offered mood or anxiety self-monitoring. In addition, one offered virtual psychotherapy (Talkspace ${ }^{31}$ ), one featured cognitive behavioral therapy (CBT)-inspired games/skills (Happify ${ }^{32}$ ), one offered support for reducing self-harm behaviors (Calm Harm ${ }^{29}$ ), and one was a health coach and behavior tracker (Fabulous: Motivate $\mathrm{Me}^{33}$ ). In most cases, total download counts were higher on Apple than on Google Play. Notable exceptions included Lumosity, $^{30}$ Fabulous: Motivate Me, ${ }^{33}$ and Daylio, ${ }^{34}$ all of which had more Google Play downloads.

Patterns of monthly (MAU) and daily active user (DAU) counts (Table 1) tended to follow total downloads within the top 25, with cognitive training and meditation apps having the highest utilization. Similar to downloads, utilization figures were higher for Apple than for Google Play. Apple had five apps with more than one million MAUs and two with more than one million DAUs. Google Play had no apps with either of those distinctions. Further, 19 of the top 25 apps had $<100,000$ Google Play MAUs, while only 2 apps had that few Apple Store MAUs.

App evaluation frameworks

As detailed in Table 2 for the top 10 apps, there was wide variability in ratings provided by the three evaluation frameworks (see Table S1 in the online supplement for ratings of the top 25 apps). No single framework evaluated all of the most downloaded apps; the Organization for the Review of Care and Health Applications (ORCHA) ${ }^{35}$ reviewed the highest number $(n=22)$ followed by PsyberGuide ${ }^{36}(n=19)$ and MindTools.io ${ }^{37}(n=14)$. There was considerable overlap in app evaluation by the three frameworks (Fig. 2). The mean age (in days) of expert reviews ranged from 109 (ORCHA) to 714 (MindTools.io), while median age (in days) ranged from 38 (ORCHA) to 776 (MindTools.io)-see Table 3 for details. No individual app received top-tercile scores for all categories across all frameworks. Fleiss' Exact Kappa scores ranged from 0.147 (Data Use \& Security) to 0.228 (Credibility \& Evidence Base), suggestive of slight to fair reliability overall (Table 4).

\section{User Experience}

Across the three frameworks, there were 54 unique expert reviews of User Experience for the top 25 apps. Of the three domains, this had the highest fraction of top (third) tercile ratings (35/54 or $64.8 \%)$. The corresponding fractions for the second and first terciles were $13 / 54(24.1 \%)$ and $6 / 54(11.1 \%)$, respectively (Table 4).

With a Fleiss' Exact Kappa score of 0.13 (slight agreement), User Experience ranked at the bottom of the three domains with regard to reliability. It was rated by at least two frameworks for 18 of the apps, with 12 having ratings from all three. Consistency was noted in the top-tercile user experience ratings of Lumosity, ${ }^{30}$ Headspace, ${ }^{38} \mathrm{Calm}^{39}{ }^{30}$ Daylio, ${ }^{34}$ and $7 \mathrm{Cups}^{40}{ }^{4}$ Seven of the top 25 apps had zero or one user experience expert review, meaning that we could not assess their degree of concordance. No app had consistently poor ratings across frameworks for this domain. Of note, ratings of user experience seemed to have the most convergence with download ranking, with most apps in the top ten rated favorably in this category. 


\begin{tabular}{|c|c|c|c|c|c|c|c|c|c|}
\hline Overall rank & App name & App type & $\begin{array}{l}\text { Overall } \\
\text { downloads }\end{array}$ & \multicolumn{3}{|c|}{ Apple App Store (iOS) } & \multicolumn{3}{|c|}{$\begin{array}{l}\text { Google Play Store } \\
\text { (Android) }\end{array}$} \\
\hline 1 & Peak—Brain Training & Cognitive training & $42 \mathrm{M}$ & $29 M$ & $4 \mathrm{M}$ & $20 \mathrm{M}$ & $13 \mathrm{M}$ & $101 \mathrm{~K}$ & $619 \mathrm{~K}$ \\
\hline 2 & Lumosity & Cognitive training & $27 \mathrm{M}$ & $12 \mathrm{M}$ & $3 \mathrm{M}$ & $9 M$ & $15 \mathrm{M}$ & $129 \mathrm{~K}$ & $790 K$ \\
\hline 5 & Relax Melodies: Sleep Sounds & Meditation & $15 \mathrm{M}$ & $9 M$ & $234 \mathrm{~K}$ & $2 \mathrm{M}$ & $6 \mathrm{M}$ & $24 K$ & $208 K$ \\
\hline 6 & Fabulous-Self Care & Health coach, behavior tracker & $6 \mathrm{M}$ & $927 \mathrm{~K}$ & $17 \mathrm{~K}$ & $165 \mathrm{~K}$ & $5 \mathrm{M}$ & $10 \mathrm{~K}$ & $71 \mathrm{~K}$ \\
\hline 7 & Daylio & Mood tracker & $6 \mathrm{M}$ & $632 \mathrm{~K}$ & $11 \mathrm{~K}$ & $103 \mathrm{~K}$ & $5 \mathrm{M}$ & $18 \mathrm{~K}$ & $158 \mathrm{~K}$ \\
\hline 8 & Insight Timer & Meditation & $5 \mathrm{M}$ & $3 \mathrm{M}$ & $51 \mathrm{~K}$ & $409 \mathrm{~K}$ & $2 \mathrm{M}$ & $8 \mathrm{~K}$ & $64 \mathrm{~K}$ \\
\hline 9 & Stop, Breathe and Think & Meditation & $4 M$ & $3 \mathrm{M}$ & $45 \mathrm{~K}$ & $414 \mathrm{~K}$ & $1 \mathrm{M}$ & $6 \mathrm{~K}$ & $53 \mathrm{~K}$ \\
\hline 12 & Happify & $\begin{array}{l}\text { Cognitive behavioral therapy } \\
\text { games, meditation }\end{array}$ & $2 \mathrm{M}$ & $2 \mathrm{M}$ & $20 \mathrm{~K}$ & $166 \mathrm{~K}$ & $263 \mathrm{~K}$ & 635 & $6 \mathrm{~K}$ \\
\hline 13 & 7 Cups: Anxiety \& Stress Chat & Peer support & $2 \mathrm{M}$ & $2 \mathrm{M}$ & $31 \mathrm{~K}$ & $278 \mathrm{~K}$ & $749 \mathrm{~K}$ & $2 \mathrm{~K}$ & $21 \mathrm{~K}$ \\
\hline 14 & Breethe - Sleep \& Meditation & Meditation & $2 \mathrm{M}$ & $2 \mathrm{M}$ & $108 \mathrm{~K}$ & $915 \mathrm{~K}$ & $33 \mathrm{~K}$ & 850 & $8 \mathrm{~K}$ \\
\hline 15 & Smiling Mind & Meditation & $2 \mathrm{M}$ & $2 \mathrm{M}$ & $45 \mathrm{~K}$ & $426 \mathrm{~K}$ & $392 \mathrm{~K}$ & $3 \mathrm{~K}$ & $35 \mathrm{~K}$ \\
\hline 16 & The Mindfulness App & Meditation & $2 \mathrm{M}$ & $1 \mathrm{M}$ & $22 \mathrm{~K}$ & $179 \mathrm{~K}$ & $777 \mathrm{~K}$ & $3 \mathrm{~K}$ & $21 \mathrm{~K}$ \\
\hline 17 & Aura: Calm Anxiety \& Sleep & Meditation & $2 \mathrm{M}$ & $1 \mathrm{M}$ & $55 \mathrm{~K}$ & $403 \mathrm{~K}$ & $331 \mathrm{~K}$ & $5 \mathrm{~K}$ & $40 \mathrm{~K}$ \\
\hline 18 & 21-Day Meditation Experience & Meditation & $2 \mathrm{M}$ & $1 \mathrm{M}$ & $13 \mathrm{~K}$ & $102 \mathrm{~K}$ & $389 \mathrm{~K}$ & 616 & $6 \mathrm{~K}$ \\
\hline 19 & Digipill: Guided Meditation & Meditation & $2 \mathrm{M}$ & $2 \mathrm{M}$ & $145 \mathrm{~K}$ & $895 \mathrm{~K}$ & $16 \mathrm{~K}$ & 4 & 86 \\
\hline 20 & $\begin{array}{l}\text { Self-Help for Anxiety } \\
\text { Management }\end{array}$ & Anxiety tracker, meditation & $1 \mathrm{M}$ & 719K & $17 \mathrm{~K}$ & $155 \mathrm{~K}$ & $348 \mathrm{~K}$ & 491 & $5 \mathrm{~K}$ \\
\hline
\end{tabular}

Credibility \& Evidence Base

Across the three frameworks, there were 46 unique expert reviews of Credibility \& Evidence Base for the top 25 apps. The fractions of apps with third- (top), second-, and first-tercile ratings were 12/46 (26.1\%), 32/46 (69.6\%), and 2/46 (4.3\%), respectively (Table 4).

This domain had the highest Fleiss' Exact Kappa score of the three (0.228 - fair agreement). It was rated by at least 2 frameworks for 14 of the apps, with 6 having ratings from all 3 . Headspace ${ }^{38}$ was the only app to receive a top-tercile rating for creditability and evidence base by all three frameworks. Six apps had consistent middle-tercile ratings, while no individual app had consistently poor ratings across frameworks for this domain. Eleven of the top 25 apps had zero or one framework rating, meaning that we could not assess their degree of reliability.

Data Use \& Security

Across the three frameworks, there were 54 unique expert reviews of Data Use \& Security for the top 25 apps. The fractions of apps with third- (top), second-, and first-tercile ratings were $14 / 54$ $(25.9 \%), 27 / 54(50.0 \%)$, and $13 / 54(24.1 \%)$, respectively. Of note, this domain had the largest share of bottom-tercile ratings (Table 4).

Date Use \& Security had the middle Fleiss' Exact Kappa score of the three domains (0.147-slight agreement). It was rated by at least two frameworks for 18 of the apps, with 11 having ratings from all three. None of the apps had universal top-tier ratings, but nine had consistent ratings in the middle- or top-tier (among at least two of the frameworks). Additionally, unique to this domain, four apps had bottom-tier ratings either consistently or in preponderance.

Independent keyword searches

As described previously, additional PrioriData ${ }^{41}$ keyword searches were conducted for highly downloaded apps meeting study inclusion and exclusion criteria that were not rated by any of the five frameworks (and therefore did not appear in this 
A.D. Carlo et al.

Table 2. Ratings of the 10 most downloaded behavioral health applications across three expert review evaluation frameworks

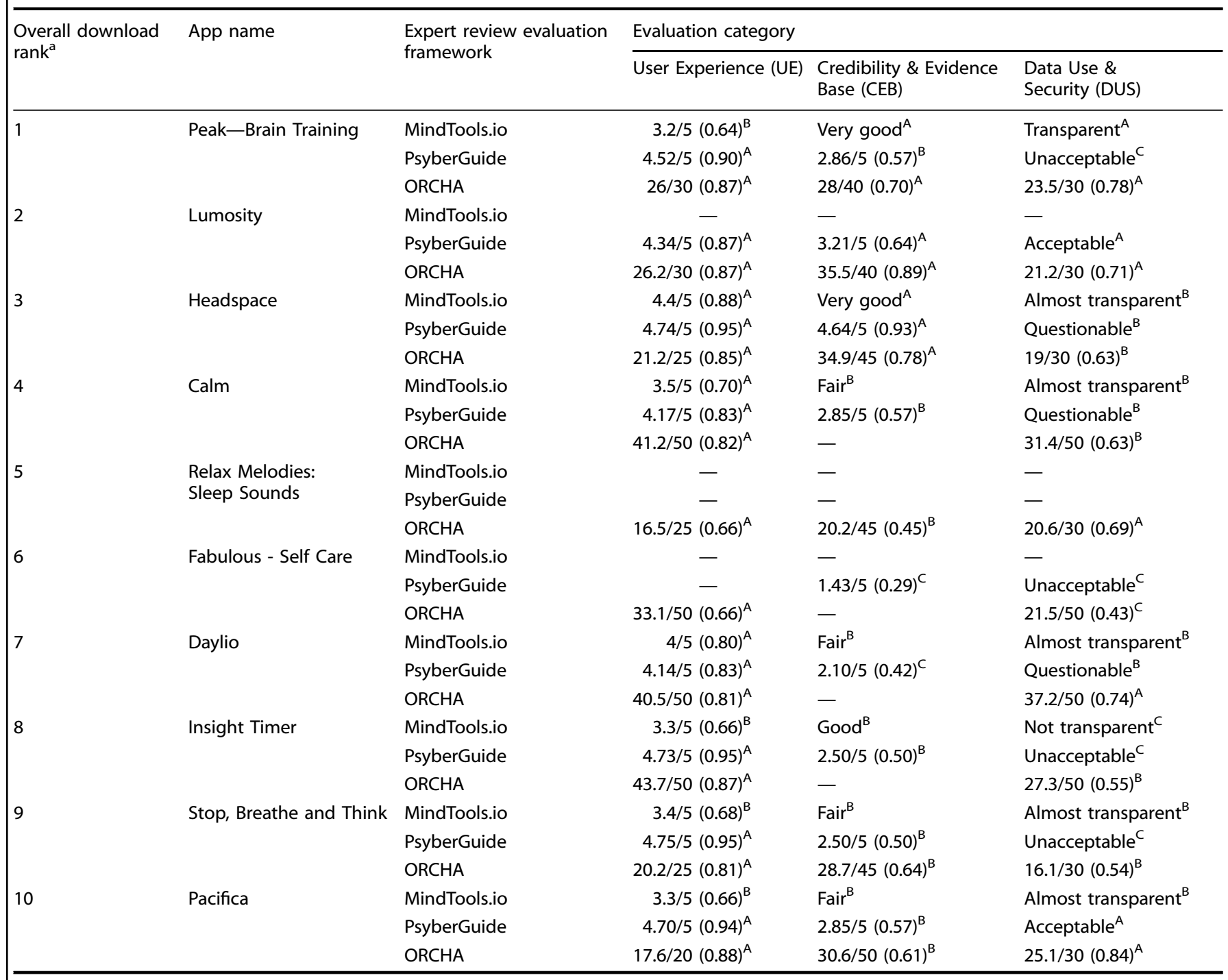

A: top-tercile rating; $\mathrm{B}$ : middle-tercile rating; $\mathrm{C}$ : bottom-tercile rating

ORCHA Organization for the Review of Care and Health Applications

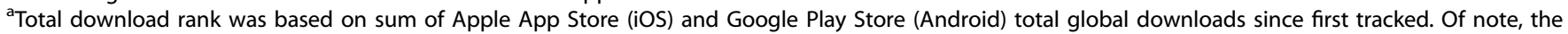
duration of time tracked by PrioriData varied across individual apps and marketplaces. The same app was often tracked over different periods of time on the Apple and Google Play App Stores

investigation's final app sample). This query yielded a total of 40 Apple and 38 Google Play apps that, if included in the final sample for this study, would have had sufficient downloads and installs to be in the 50 most popular in their respective marketplace. These represent estimates of the counts of popular behavioral health apps that were "missed" by using a rating framework-derived sampling strategy.

\section{DISCUSSION}

Our key findings detail the 25 most downloaded behavioral health apps and their corresponding evaluations across online rating frameworks with expert reviews. The issue of how to best evaluate behavioral health apps and disseminate the results is timely and significant. Although this investigation focuses on behavioral health, its methodology and findings are relevant to all of digital health. Psyberguide, ${ }^{36}$ Mintools.io, ${ }^{37}$ and $\mathrm{ORCHA}^{35}$ represent three largely transparent attempts to objectively review behavioral health apps across key domains, such as user experience, credibility, evidence base, data privacy, and security. ${ }^{24}$ Our results show that their ratings are broadly inconsistent and often contradictory, with even the most popular behavioral health apps (as determined by total global downloads) not receiving uniformly favorable scores. Quantitatively, we demonstrated that Fleiss' Exact Kappa scores for the three domains ranged from 0.147 (Data Use \& Security) to 0.228 (Credibility \& Evidence Base), suggestive of only slight to fair reliability overall. This could, in part, be a consequence of the frameworks' inclusion of inherently subjective and poorly reliable categories like User Experience, ${ }^{42}$ instead of more general and measurable consumer preferences. ${ }^{17}$ At the same time, our findings also noted significant discordance in more objective categories like Data Use \& Security, suggesting that inconsistencies are multifactorial and cannot be ascribed solely to fundamental domain characteristics. Ultimately, inter-framework discrepancies are most likely a consequence of the current lack of consensus domains and standards for behavioral health apps. 


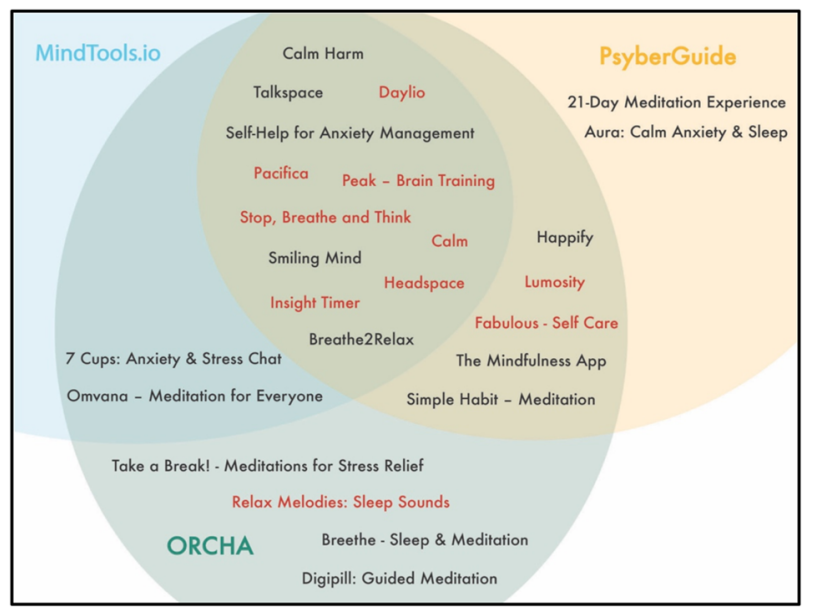

Fig. 2 Visualization of framework reach and overlap apps colored in red are in the top ten with regard to total global downloads since first tracked

\begin{tabular}{|c|c|c|c|c|}
\hline & & Mindtools.io & Psyberguide & ORCHA \\
\hline \multicolumn{2}{|c|}{ Fraction (\%) of top 25 apps reviewed } & $14 / 25(56)$ & 19/25 (76) & $22 / 25(88)$ \\
\hline \multicolumn{2}{|c|}{$\begin{array}{l}\text { Website total global visits over 3-month } \\
\text { period }^{\mathrm{a}}\end{array}$} & 22,780 & 68,041 & 40,527 \\
\hline \multirow{3}{*}{$\begin{array}{l}\text { Average age } \\
\text { of review (days) }\end{array}$} & Mean & 714 & 598 & 109 \\
\hline & Median & 776 & 424 & 38 \\
\hline & Standard deviation & 178 & 305 & 170 \\
\hline \multicolumn{5}{|c|}{$\begin{array}{l}\text { ORCHA Organization for the Review of Care and Health Applications } \\
\text { a Totals are for the 3-month period between November } 2018 \text { and January } \\
2019 \text {. Visitors may be counted more than once. Statistics powered by } \\
\text { SimilarWeb } \\
\text { b Average age of review was calculated as the mean number of days from } \\
15 \text { April } 2019\end{array}$} \\
\hline
\end{tabular}

Even with such standards, however, it remains an open question whether frameworks could keep mobile health app evaluations up-to-date and effectively disseminate them to the general public longitudinally. Of the three examined frameworks, ORCHA reviewed the largest percentage of the 25 most downloaded apps $(88 \%)$, indicating that it may be most in line with the public's revealed preferences (as measured by app downloads). Other frameworks, by this measure, were less up-to-date, with corresponding figures for Psyberguide and Mindtools.io being $76 \%$ and $56 \%$, respectively. ORCHA was also the most current for the top 25 apps, with a mean age of expert review of 109 days, less than one-fifth that of the second most current framework, Psyberguide. Given the expected continuation of rapid growth in the behavioral health app field, the challenge of keeping expert reviews up-to-date will only grow more arduous over time, raising questions about the maximum potential impact of online behavioral health app rating frameworks on public knowledge and decision-making. In fact, according to the authors' SimilarWeb-powered analysis of web traffic data, ${ }^{43}$ the most accessed of the three online frameworks, Psyberguide, was visited by a total of 68,041 users between November 2018 and January 2019. When this is juxtaposed against Peak-Brain Training's 42 million downloads, $>300,000$ Google Play reviews, and $>85,000$ Apple reviews, it appears that many customers may be seeking guidance from sources outside of the rating frameworks.

Although this does not discount the potential benefits of having objective, expert reviewers for behavioral health apps, it suggests that other strategies, such as data crowd sourcing or citizen science, ${ }^{44}$ may be more scalable, effective, and sustainable. It also suggests that frameworks designed to facilitate shared app decision-making, such as the American Psychiatric Association's (APA) app evaluation model ${ }^{45}$ and others, ${ }^{2,23}$ may be valuable tools for consumers, clinicians, and patients without providing ongoing expert reviews.

Despite notable limitations, there is valuable information to be gleaned from the app rating and ranking frameworks in this investigation, all of which have attempted to organize and present complex information in a succinct and comprehensible manner to the general public. This is particularly true in cases of concurrence across frameworks. For example, irrespective of framework, only $26 \%(12 / 46)$ of the ratings of Credibility \& Evidence Base for the top 25 apps were top-tercile. This finding is alarming, but also unsurprising, given the well-described lack of rigorous research on behavioral health and other health-related apps. ${ }^{46}$ It does not, however, appear to stop developers from marketing apps as either standalone or adjunctive treatments for common behavioral health disorders (e.g., depression, anxiety); let the buyer beware of such tools in the absence of universal standards. Similarly, limited transparency around Data Use \& Security led 24\% (13/54) of the ratings to be bottom-tercile, the highest percentage of the three domains. The need for data privacy in the digital age is paramount, yet a lack of transparency and standardization remains prevalent in the marketplace. ${ }^{47}$

In addition to demonstrating that popular behavioral health apps may have poor or questionable support from the literature, the results of this investigation also showed that the most commonly downloaded apps (and also those with some of the highest counts of DAUs and MAUs) simply offered relaxation, meditation, or mindfulness skills, rather than bona fide behavioral health treatments. These frequently downloaded and used apps have defied the odds, as research has demonstrated that many users stop accessing behavioral health digital interventions within 2 weeks of the initial download. ${ }^{48}$ This poses an important question-what role do consumers feel that mobile technology should play in behavioral health recovery? As a recently published survey uncovered, most people who would consider using appbased care interventions were skeptical of completely self-guided tools. ${ }^{49}$ This could signify that behavioral health apps are most suitable for tracking or mindfulness and that evidence-based treatments (e.g., psychotherapy or brief interventions) are best reserved for traditional care. It also suggests that academic researchers and clinicians designing and evaluating apps may be missing what consumers are actually seeking. If researchers do aim to make mobile health interventions with evidence-based treatments attractive and accessible in the "real world," they should be sure to focus on subjective constructs (e.g., user experience) early in the design phase and ensure that their apps are available on the Apple and Google Play marketplaces. Owing to the requirement of diverse skillsets and expertise, it is likely that successful promotions of evidence-based behavioral health apps will require ongoing, meaningful collaborations between clinicians, thought leaders, and digital user experience professionals.

Although rigorous sampling methods were employed in this investigation, our final sample was limited by the content of the five included frameworks. This largely restricted our focus to English language apps, despite our lack of specific exclusion criteria for apps unavailable in English. Our finding of additional Apple (iOS) and Google Play (Android) apps through PrioriData ${ }^{41}$ keyword searches demonstrated that a number of popular apps were "missed" using this strategy. The "missing" apps were either unrated by popular rating frameworks for unknown reasons or had not yet been rated at the time of the study. The use of a framework-derived sampling strategy, however, allowed the authors to assess the degree of concordance between highly visible, consumer-facing frameworks, which was a primary 
A.D. Carlo et al.

Table 4. Rating concordance and tercile distribution of domains across frameworks

\begin{tabular}{|c|c|c|c|c|c|}
\hline User Experience (UE) & & Credibility \& Evidence Base (CEB & & Data Use \& Security (DUS) & \\
\hline $\begin{array}{l}\text { Fractions of expert reviews by } \\
\text { tercile }\end{array}$ & $\begin{array}{c}35 / 54(0.65)^{A} \\
13 / 54(0.24)^{B} \\
6 / 54(0.11)^{C}\end{array}$ & $\begin{array}{l}\text { Fractions of expert reviews by } \\
\text { tercile }\end{array}$ & $\begin{array}{c}12 / 46(0.26)^{\mathrm{A}} \\
32 / 46(0.70)^{\mathrm{B}} \\
2 / 46(0.043)^{\mathrm{C}}\end{array}$ & $\begin{array}{l}\text { Fractions of expert reviews by } \\
\text { tercile }\end{array}$ & $\begin{array}{l}14 / 54(0.26)^{A} \\
27 / 54(0.50)^{B} \\
13 / 54(0.24)^{C}\end{array}$ \\
\hline
\end{tabular}

objective of this investigation. Further, our sampling design forced exclusion of apps that were either (1) available only through a web browser or (2) unavailable on the Apple or Google Play marketplaces. This was necessary because PrioirData ${ }^{41}$ could not provide download or utilization data on apps that were not available through one of the two primary marketplaces. Of note, it is almost certain that any sampling technique would have missed large numbers of apps, given the $>10,000$ that are thought to be available for behavioral health. Additionally, this investigation was limited by the constraints of our data source. Although PrioriData $^{41}$ uses rigorous methodology to obtain its estimates of total downloads, DAUs, and MAUs, these results are largely model derived and are subject to statistical error margins (which were not available to the authors for this investigation). Finally, for total downloads, all behavioral health apps were tracked for different periods of time on PrioriData. ${ }^{41}$ Identical apps on Apple and Google Play were also tracked for different periods of time, depending on when the app was made available by the developer on the particular marketplace. However, the primary purpose of this investigation was to provide an estimate of total counts of unique downloads in the "real world," as opposed to providing a download rate over time.

Although the behavioral health app market has grown rapidly in recent years, it has been minimally evaluated or regulated and little is known about "real world" usage patterns. The results of this investigation demonstrate that the most commonly downloaded and installed behavioral health apps tend to focus on relaxation, mindfulness, and meditation, as opposed to bona fide treatments. In addition, findings suggest that consumer-facing behavioral health app rating and ranking frameworks fail to score a number of popular apps and are often discordant in their existing evaluations. Finally, there is a clear gap between behavioral health research and consumers' revealed preferences. Successful promotions of evidence-based apps will likely require ongoing, meaningful collaborations between clinicians, thought leaders, and digital user experience professionals.

\section{METHODS}

Behavioral health app sample

The authors obtained the study sample of mobile (smartphone- or tabletbased) behavioral health apps from five consumer-facing, publicly available online rating frameworks with expert reviews ${ }^{35-37,50,51}$ identified in recently published literature. ${ }^{5,23,52}$ One framework (AppScript ${ }^{53}$ ) was excluded, as it was primarily designed for health providers (e.g., physicians, nurses, health coaches) instead of patients or general consumers. Three of the five included frameworks rated only behavioral health apps, ${ }^{36,37,51}$ while the other two rated health apps of all types. ${ }^{35,50}$ All apps from the three exclusively behavioral health frameworks (Psyberguide, ${ }^{36}$ Mindtools. $\mathrm{io}^{37}$ and the Anxiety and Depression Association of America (ADAA) $)^{51}$ ) were included in the study sample. For $\mathrm{ORCHA},{ }^{35}$ all apps flagged from keyword searches for "Stress and Anxiety" and "Depression" were included. For RankedHealth, ${ }^{50}$ all apps were manually reviewed and included if there was any clear, broadly defined association with mental or behavioral health. Duplicate apps were removed from the final study sample.
Apps were excluded from the final sample if they were: (1) functional only through a web browser interface (because our market research firm's (PrioriData ${ }^{41}$ ) download/installation and utilization estimates are partially derived from mobile app marketplaces), (2) no longer on the market, and (3) not designed to treat a behavioral health disorder or not promoting a specific behavioral health treatment or technique (e.g., mindfulness, meditation). To exemplify this third criterion, apps for sleep were included only if they included a specific behavioral health treatment or technique, such as CBT for insomnia. ${ }^{54}$ This excluded apps that promoted sleep through digital alarm clocks, "trackers," or other methods. In addition, apps whose primary focus was not a behavioral health technique (e.g., imagery apps, white noise apps, or health and wellness apps) were excluded, although some behavioral health-focused apps did include these features as part of their treatment packages. Decisions to include/exclude apps were made independently by two members of the research team (A.D.C., R. H.G., or B.N.R.). Discrepant decisions were discussed during team meetings and revisions were made accordingly.

Market research data-PrioriData ${ }^{41}$

Download, installation, and utilization data for all behavioral health apps were procured from PrioriData, ${ }^{41}$ a leading mobile app market research firm based in Berlin, Germany that has been cited in previous literature. ${ }^{55-}$ ${ }^{57}$ PrioriData ${ }^{41}$ uses publicly available data, proprietary data (from strategic partners), and modeling techniques to estimate download and utilization rates for mobile apps worldwide. ${ }^{41}$ Each day, PrioriData ${ }^{41}$ obtains actual event, demographic, location, device, and installed app data from $>3$ billion unique-user devices across $>100$ countries. ${ }^{41}$ When applicable, data obtained for this investigation were available for all apps from the Apple and Google Play Stores, the two leading marketplaces worldwide. The authors had access to PrioriData's ${ }^{41}$ platform between 21 September and 21 October 2018. All download and utilization data for this investigation were obtained over the course of this 30-day period.

Separately for the Apple and Google Play marketplaces, PrioriData ${ }^{41}$ provided the following global estimates for all included study apps: (1) total downloads since first tracked, (2) DAUs from the past 30 days, and (3) MAUs from the past 30 days. Of note, for total global downloads, all apps were not tracked for the same amount of time. The "first tracked" date corresponds to the time point at which PrioriData ${ }^{41}$ was able to detect and estimate data for an app. This time point typically occurs shortly after an app is available on the Apple or Google Play marketplace. Finally, the authors ran app keyword searches for the following eight terms —-"Depression," "Anxiety," "Mood Disorder," "Mental Health," "Behavioral Health," "Psychiatry," "Psychology," and "Stress." Each was queried for total downloads, DAUs, and MAUs. The top 500 results from each query were downloaded. The intention of these keyword searches was to identify behavioral health apps that were not rated in any of the five aforementioned frameworks.

Association of download, installation, and utilization data with framework ratings

After data were procured for all apps and appropriate exclusions were made from the sample, the authors summed the total Apple and Google Play global downloads (since first tracked) for all apps. For the top 25 apps by combined total downloads, ratings were compared across the Psyberguide, ${ }^{36}$ Mintools.io, ${ }^{37}$ and $\mathrm{ORCHA}^{35}$ frameworks. These three frameworks were chosen because they each rated a substantial number of behavioral health apps and are public-facing tools meant to appeal to consumers, clinicians, and researchers. Each framework was different in its 
structure, methodology, and terminology, with details available online. Psyberguide ${ }^{36}$ (https://psyberguide.org/about-psyberguide/) and Mintools. io $^{37}$ (https://mindtools.io/mindtools-io-scoring/) each consistently had three rating categories, while $\mathrm{ORCHA}^{35}$ (https://www.orcha.co.uk/oursolution/the-orcha-review/\#0) varied between having three and four. The authors did not include or consider ORCHA's ${ }^{35}$ fourth category (overall score), as Psyberguide ${ }^{36}$ and Mintools.io ${ }^{37}$ did not have a corresponding category. Although each framework had its own category labels, they all assessed analogous app characteristics and constructs. This made it possible for us to group similar categories together for ease of comparison across frameworks. Program Quality (MindTools.io ${ }^{37}$ ) and User Experience (PsyberGuide, ${ }^{36} \mathrm{ORCHA}^{35}$ ) were grouped under the "User Experience" category. Source Credibility (MindTools.io ${ }^{37}$ ), Credibility (PsyberGuide ${ }^{36}$ ), and Clinical Assurance $\left(\mathrm{ORCHA}^{35}\right)$ were grouped under "Credibility \& Evidence Base." Finally, Privacy Explanation (MindTools.io ${ }^{37}$ ), Transparency $\left(\right.$ PsyberGuide $\left.{ }^{36}\right)$, and Data Security $\left(\mathrm{ORCHA}^{35}\right)$ were grouped as "Data Use \& Security."

The three frameworks also differed in the presentation of their data, with some electing to provide numeric or fractional scores and others using qualitative scores. Even within categories of the same framework, data presentation varied significantly at times. For example, within $\mathrm{ORCHA}{ }^{35}$ rating categories, fractional scores were occasionally presented with different denominators. Whenever possible, fractional scores were converted to decimals for consistency and ease of comparison.

Terciles were determined differently for each framework and metric. In its detailed methodology, Mindtools.io ${ }^{37}$ provides a key for interpretation of each score. This was used to determine terciles for all Mindtools.io ${ }^{37}$ categories. Of note, in categories with more than three score levels (either quantitative or qualitative), terciles were not able to be matched one-toone. ORCHA ${ }^{35}$ publishes three-level interpretative ranges (green, yellow, and red) for ratings that are based on the percentage score for each category or domain. To best maintain the integrity of the $\mathrm{ORCHA} \mathrm{A}^{35}$ rating system, these score ranges were maintained and directly used to inform tercile assignment (i.e., green scores from ORCHA were considered toptercile in this study). In cases where ORCHA provided more than one evaluation of the same app (e.g., if more than one release was evaluated and both remained visible on the website), the newest app version or latest review was used. Since Psyberguide ${ }^{36}$ does not provide clear interpretive guidance for comparison of its composite quantitative scores, tercile ranges were calculated from the distribution of all available scores and each app was assigned accordingly. For Psyberguide's $s^{36}$ three-level qualitative metric (transparency), terciles were matched one-to-one. To assess the reliability of agreement between frameworks, the authors calculated Fleiss' Exact Kappa for each individual rating domain. Fleiss' Exact Kappa $(\kappa)$ is an index of agreement for more than two raters that is adjusted for chance. ${ }^{58}$ Although there is no universal interpretive scale, the following is frequency used: $<0$ (poor agreement), $0.0-0.20$ (slight agreement), $0.21-0.40$ (fair agreement), $0.41-0.60$ (moderate agreement), 0.61-0.80 (substantial agreement), and 0.81-1.0 (almost perfect agreement). ${ }^{59}$ All calculations were conducted using Microsoft Excel and R's "irr" package. ${ }^{60}$ Additionally, the authors used SimilarWeb ${ }^{43}$ to analyze web traffic data (including total users) for all three frameworks between November 2018 and January 2019.

Finally, the authors calculated how commonly an app appeared across frameworks and how up-to-date each framework was (timeliness). We calculated the percentage of top 25 apps that were rated by each framework, and the mean difference (in days) between the most recent online expert review for each top 25 app and 15 April 2019. Of note, Mindtools.io, ${ }^{37}$ Psyberguide, ${ }^{36}$ and $\mathrm{ORCHA}{ }^{35}$ publish their date of review, while RankedHealth ${ }^{50}$ and $A D A A^{51}$ do not. For frameworks that provided month and year only for expert reviews, the date of review was assumed to be the 15th day of the month.

This protocol was reviewed and granted exemption by the University of Washington Human Subjects Division (Protocol \#: 5714).

\section{Reporting summary}

Further information on research design is available in the Nature Research Reporting Summary linked to this article.

\section{DATA AVAILABILITY}

The data generated during and/or analyzed in this study are available from adc42@uw.edu on reasonable request.

\section{ACKNOWLEDGEMENTS}

We acknowledge the contribution of Anisha Keshavan, $\mathrm{PhD}$ of the Institute for Learning \& Brain Sciences at University of Washington for her partnership, expertise, and guidance. We also acknowledge Emily Friedman MID, CPE of the University of Washington for her help with data visualization. This work was supported by the National Institute of Mental Health (T32MH073553, P50MH115837, R25MH104159) and the VA Advanced Fellowship Program in Parkinson's Disease.

\section{AUTHOR CONTRIBUTIONS}

Guarantors: All authors had full access to the study data and take responsibility for the integrity of the complete work and the final decision to submit the manuscript. Study concept and design: All. Acquisition, analysis, or interpretation of data: All. Drafting of the manuscript: A.D.C., B.N.R., R.H.G. Critical revision of the manuscript: All. Obtaining fund: R.H.G. and P.A.A.

\section{ADDITIONAL INFORMATION}

Supplementary information accompanies the paper on the npj Digital Medicine website (https://doi.org/10.1038/s41746-019-0129-6).

Competing interests: The authors declare no competing interests.

Publisher's note: Springer Nature remains neutral with regard to jurisdictional claims in published maps and institutional affiliations.

\section{REFERENCES}

1. Aitken, M. \& Lyle, J. Patient Adoption of mHealth - Use, Evidence and Remaining Barriers to Mainstream Acceptance (IMS Institute for Healthcare Informatics, Parsippany, NJ, 2015).

2. Neary, M. \& Schueller, S. M. State of the field of mental health apps. Cogn. Behav. Pract. 25, 531 (2018).

3. Pohl, M. 325,000 mobile health apps available in 2017 - Android now the leading mHealth platform. https://research2guidance.com/325000-mobile-health-appsavailable-in-2017/ (2017).

4. Torous, J. B., Chan, S. R., Yellowlees, P. M. \& Boland, R. To use or not? Evaluating ASPECTS of smartphone apps and mobile technology for clinical care in psychiatry. J. Clin. Psychiatry 77, 734-738 (2016).

5. Torous, J. et al. The emerging imperative for a consensus approach toward the rating and clinical recommendation of mental health apps. J. Nerv. Ment. Dis. 206, 662-666 (2018)

6. Kuester, A., Niemeyer, H. \& Knaevelsrud, C. Internet-based interventions for posttraumatic stress: a meta-analysis of randomized controlled trials. Clin. Psychol. Rev. 43, 1-16 (2016).

7. Mohr, D. C., Weingardt, K. R., Reddy, M. \& Schueller, S. M. Three problems with current digital mental health research... and three things we can do about them. Psychiatr. Serv. 68, 427-429 (2017)

8. Richards, D. \& Richardson, T. Computer-based psychological treatments for depression: a systematic review and meta-analysis. Clin. Psychol. Rev. 32, 329-342 (2012).

9. Anthes, E. Pocket psychiatry - mobile mental-health apps have exploded onto the market, but few have been thoroughly tested. Nature 532, 20-23 (2016).

10. Gilbody, S. et al. Computerised cognitive behaviour therapy (cCBT) as treatment for depression in primary care (REEACT trial): large scale pragmatic randomised controlled trial. BMJ 351, h5627 (2015).

11. Arean, P. A. et al. The use and effectiveness of mobile apps for depression: results from a fully remote clinical trial. J. Med. Internet Res. 18, e330 (2016).

12. Baumel, A. \& Yom-Tov, E. Predicting user adherence to behavioral eHealth interventions in the real world: examining which aspects of intervention design matter most. Transl. Behav. Med 8, 793-798 (2018).

13. Kohl, L. F., Crutzen, R. \& de Vries, N. K. Online prevention aimed at lifestyle behaviors: a systematic review of reviews. J. Med Internet Res 15, e146 (2013).

14. Lee, J. A. et al. Attitudes and preferences on the use of mobile health technology and health games for self-management: interviews with older adults on anticoagulation therapy. JMIR Mhealth Uhealth 2, e32 (2014).

15. Rosa, C., Campbell, A. N., Miele, G. M., Brunner, M. \& Winstanley, E. L. Using etechnologies in clinical trials. Conte. Clin. Trials 45, 41-54 (2015).

16. Torous, J., Levin, M. E., Ahern, D. K. \& Oser, M. L. Cognitive behavioral mobile applications: clinical studies, marketplace overview, and research agenda. Cogn. Behav. Pract. 24, 215-225 (2017).

17. Wisniewski, $H$. et al. Understanding the quality, effectiveness and attributes of top- rated smartphone health apps. Evid Based Mental Health https://doi.org/ 10.1136/ebmental-2018-300069 (2019). 
18. Krebs, P. \& Duncan, D. T. Health app use among US mobile phone owners: a national survey. JMIR Mhealth Uhealth 3, e101 (2015).

19. Huang, H. Y. \& Bashir, M. Users' adoption of mental health apps: examining the impact of information cues. JMIR Mhealth Uhealth 5, e83 (2017).

20. American Psychiatric Association (APA). App evaluation model. https://www. psychiatry.org/psychiatrists/practice/mental-health-apps/app-evaluation-model (2018).

21. Baumel, A., Faber, K., Mathur, N., Kane, J. M. \& Muench, F. Enlight: a comprehensive quality and therapeutic potential evaluation tool for mobile and webbased eHealth interventions. J. Med. Internet Res. 19, e82 (2017).

22. Schueller, S. Should you trust mental health apps? http://www. theneuroethicsblog.com/2018/05/should-you-trust-mental-health-apps.html (2018).

23. Moshi, M. R., Tooher, R. \& Merlin, T. Suitability of current evaluation frameworks for use in the health technology assessment of mobile medical applications: a systematic review. Int. J. Technol. Assess. Health Care 34, 464-475 (2018).

24. Torous, J. et al. Towards a consensus around standards for smartphone apps and digital mental health. World Psychiatry 18, 97-98 (2019).

25. National Institute of Mental Health. Technology and the future of mental health treatment. https://www.nimh.nih.gov/health/topics/technology-and-the-futureof-mental-health-treatment/index.shtml (2018).

26. United States Food and Drug Administration. Digital Health Software Precertification (Pre-Cert) Program. https://www.fda.gov/medicaldevices/ digitalhealth/digitalhealthprecertprogram/default.htm (2019).

27. World Health Organization. Classification of Digital Health Interventions v1.0. 1-20 (2018).

28. Peak-Brain Training. Peak-Brain Training. http://www.peak.net (2019).

29. Stem4 - stemming teenage mental illness; supporting teenage mental health. Calm Harm. https://stem4.org.uk (2019).

30. Lumosity. https://www.lumosity.com/en/ (2019).

31. Talkspace. https://www.talkspace.com (2019).

32. Happify. https://www.happify.com (2019).

33. Fabulous. https://thefabulous.co/ (2019).

34. Daylio. https://daylio.webflow.io (2019).

35. Organization for the Review of Care and Health Applications (ORCHA). https:// www.orcha.co.uk (2019).

36. Psyberguide. Psyberguide - A Project of One Mind. https://psyberguide.org (2019).

37. Mindtools.io. https://mindtools.io/find-a-program/ (2019).

38. Headspace. https://www.headspace.com (2019).

39. Calm. https://www.calm.com (2019).

40. 7 Cups. 7 Cups of Tea. https://www.7cups.com (2019).

41. PrioriData. https://prioridata.com (2019).

42. Ng, M. M., Firth, J., Minen, M. \& Torous, J. User engagement in mental health apps: a review of measurement, reporting, and validity. Psychiatr. Serv. appips201800519 (2019).

43. SimilarWeb. https://www.similarweb.com (2019).

44. Wazny, K. Applications of crowdsourcing in health: an overview. J. Glob. Health 8 , 010502 (2018).

45. American Psychiatric Association (APA). App Evaluation Model. https://www. psychiatry.org/psychiatrists/practice/mental-health-apps/app-evaluation-model (2019).
46. Safavi, K., Mathews, S. C., Bates, D. W., Dorsey, E. R. \& Cohen, A. B. Top-funded digital health companies and their impact on high-burden, high-cost conditions. Health Affairs (Millwood) 38, 115-123 (2019).

47. Huckvale, K., Torous, J. \& Larsen, M. E. Assessment of the data sharing and privacy practices of smartphone apps for depression and smoking cessation. JAMA Netw. Open 2, e192542 (2019).

48. Dorsey, E. R. et al. The use of smartphones for health research. Acad. Med. 92, 157-160 (2017).

49. Renn, B. N., Hoeft, T. J., Lee, H. S., Bauer, A. M. \& Areán, P. A. Preference for inperson psychotherapy versus digital psychotherapy options for depression: survey of adults in the U.S. npj Digital Med. 2, https://doi.org/10.1038/s41746-0190077-1 (2019).

50. RankedHealth. Curated health apps \& devices - with a focus on clinical relevance, safety, and efficacy. http://www.rankedhealth.com (2019).

51. Anxiety and Depression Association of America. ADAA Reviewed Mental Health Apps. https://adaa.org/finding-help/mobile-apps (2019).

52. Neary, M. \& Schueller, S. State of the field of mental health apps. Cognitive and Behavioral Practice. 25, 531-537. https://doi.org/10.1016/j.cbpra.2018.01.002 (2018).

53. IQVIA. AppScript. https://www.appscript.net/ (2019).

54. Edinger, J. D., Wohlgemuth, W. K., Radtke, R. A., Marsh, G. R. \& Quillian, R. E. Cognitive behavioral therapy for treatment of chronic primary insomnia. JAMA 285, https://doi.org/10.1001/jama.285.14.1856 (2001).

55. Williams, G. \& Mahmoud, A. Modeling user concerns in the app store: a case study on the rise and fall of yik yak. In 2018 IEEE 26th International Requirements Engineering Conference (RE) 64-75 (IEEE, 2018).

56. Boudreau, K., Jeppesen, L. B. \& Miric, M. Competing on free(mium): digital competition with network effects. SSRN Electron. J. https://doi.org/10.2139/ ssrn.2984546 (2017).

57. Comino, S., Manenti, F. M. \& Mariuzzo, F. Updates management in mobile applications: iTunes vs Google Play. J. Econ. Manag. Strategy https://doi.org/ 10.1111/jems.12288 (2018).

58. Falotico, R. \& Quatto, P. Fleiss' kappa statistic without paradoxes. Qual. Quant. 49, 463-470 (2014).

59. Landis, J. R. \& Koch, G. G. The measurement of observer agreement for categorical data. Biometrics 33, 159-174 (1977).

60. Gamer, M. Package 'irr'. https://cran.r-project.org/web/packages/irr/irr.pdf (2019).

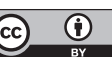

Open Access This article is licensed under a Creative Commons Attribution 4.0 International License, which permits use, sharing, adaptation, distribution and reproduction in any medium or format, as long as you give appropriate credit to the original author(s) and the source, provide a link to the Creative Commons license, and indicate if changes were made. The images or other third party material in this article are included in the article's Creative Commons license, unless indicated otherwise in a credit line to the material. If material is not included in the article's Creative Commons license and your intended use is not permitted by statutory regulation or exceeds the permitted use, you will need to obtain permission directly from the copyright holder. To view a copy of this license, visit http://creativecommons. org/licenses/by/4.0/.

(c) The Author(s) 2019 